INPLASY

PROTOCOL

To cite: Guo et al.

Effectiveness and Safety of

Massage for Athletic Injuries: a

Protocol for Systematic

Review and Meta-analysis.

Inplasy protocol 202170066.

doi:

10.37766/inplasy2021.7.0066

Received: 20 July 2021

Published: 20 July 2021

Corresponding author:

Min Fang

fm-tn0510@shutcm.edu.cn

Author Affiliation:

Shanghai University of

Traditional Chinese Medicine.

Support: University.

Review Stage at time of this submission: The review has not yet started.

Conflicts of interest:

None declared.

\section{Effectiveness and Safety of Massage for Athletic Injuries: a Protocol for Systematic Review and Meta-analysis}

Guo, G1; Xie, S2; Cai, F3; Zhou, X4; Xu, J5; Wu, B6; Wu, G7; Xiao, $\mathrm{R}^{8} ; \mathrm{Xu}, \mathrm{X}^{9}$; Lu, $\mathrm{P}^{10}$; Fang, $\mathrm{M}^{11}$.

Review question / Objective: The aim of this protocol for systematic review and meta-analysis of randomized controlled trials is to evaluate the efficacy and safety of massage therapy for athletic injuries.

Condition being studied: Athletic injuries. Massage Therapy. Information sources: A systematic search will be performed in the following electronic databases for randomized controlled trials (RCTs) to evaluate the effectiveness and safety of massage therapy in treating athletic injuries: PubMed, the Cochrane Library, EMBASE and four Chinese databases (CNKI, Wan Fang, CBMdisc and VIP). Each database will be searched from inception to July 2021.

INPLASY registration number: This protocol was registered with the International Platform of Registered Systematic Review and Meta-Analysis Protocols (INPLASY) on 20 July 2021 and was last updated on 20 July 2021 (registration number INPLASY202170066).

\section{INTRODUCTION}

Review question / Objective: The aim of this protocol for systematic review and meta-analysis of randomized controlled trials is to evaluate the efficacy and safety of massage therapy for athletic injuries.

Condition being studied: Athletic injuries. Massage Therapy.

\section{METHODS}

Participant or population: Inclusion criteria: Adults with athletic injuries (as diagnosed by a clinician, or using any recognized diagnostic criteria) will be included. Exclusion criteria: Severe primary disease such as cardiovascular, lung, kidney, and hematopoietic disease, pregnant woman, patients with mental illness. 
Intervention: Massage was the main intervention (e.g. Chinese massage, and Thai massage).

Comparator: The interventions of control group will involve any therapy other than massage (e.g., medication, placebo, routine care, etc.).

Study designs to be included: We will include randomised trials to assess the beneficial effects of the treatments, and will supplement these with observational studies for the assessment of harms.

Eligibility criteria: Only RCTs about massage for athletic injuries will be included, with language restrictions in English or Chinese. Case report, experience report, and laboratory studies will not be included.

Information sources: A systematic search will be performed in the following electronic databases for randomized controlled trials (RCTs) to evaluate the effectiveness and safety of massage therapy in treating athletic injuries: PubMed, the Cochrane Library, EMBASE and four Chinese databases (CNKI, Wan Fang, CBMdisc and VIP). Each database will be searched from inception to July 2021.

Main outcome(s): Change in pain score from baseline to the last available followup, measured using the Visual Analogue Scale (VAS).

Quality assessment / Risk of bias analysis: The risk of bias for each of the following domains will be assessed for each study: (1) random sequence generation, (2) allocation concealment, (3) blinding of participants and personnel, (4) blinding of outcome assessments, (5) incomplete outcome data, (6) selective reporting, and (7) other bias. Each study included will be rated as having a high, low, or unclear risk of bias. Two reviewers (SX and FC) will evaluate the consistency of all the extracted data and quality ratings. Disagreements will be resolved by discussion with a third reviewer (GG).
Strategy of data synthesis: For discontinuous variables, the risk ratio (RR) with $95 \%$ confidence interval $(\mathrm{Cl})$ will be selected. For continuous variables, the weighted mean difference (WMD) with 95\% $\mathrm{Cl}$ will be selected when the measuring instruments are the same, and the standardized mean difference (SMD) with $95 \% \mathrm{Cl}$ will be selected when the measuring instruments are different. We will use the fixed-effect model if there is no significant heterogeneity $(\mathrm{P}>\mathrm{.} \mathbf{1}$ or $\mathbf{1 2} .1$ or $\mathbf{I} 2<$ $50 \%$ ), we will conduct subgroup analysis or sensitivity analysis to identify possible causes of heterogeneity among populations.

Subgroup analysis: If the necessary data are available, subgroup analysis will be conducted according to the following criteria : (1)The treatment period. (2)Different acupuncture points with massage. (3)Different types of manipulation (e.g., kneading, rolling, pressing).

Sensitivity analysis: To identify the robustness of the meta-analysis, lowquality trials, with high risks of bias or outcomes that are seriously distant from the rest of the data, will be excluded.

Country(ies) involved: China.

Keywords: Athletic Injuries, Massage, Protocol, Systematic Review, Metaanalysis.

Contributions of each author:

Author 1 - Guangxin Guo.

Author 2 - Shengji Xie.

Author 3 - Feihong Cai.

Author 4 - Xu Zhou.

Author 5 - Jianghan Xu.

Author 6 - Boyi Wu.

Author 7 - Guanghui Wu.

Author 8 - Ran Xiao.

Author 9 - Xiruo Xu.

Author 10 - Ping Lu.

Author 11 - Min Fang. 